\title{
Determination of Strong Acidic Drugs in Biological Matrices: A Review of Separation Methods
}

\author{
Lingli Mu, ${ }^{1}$ Feifan Xie, ${ }^{1,2}$ Sanwang Li, ${ }^{2}$ and Peng $\mathrm{Yu}^{2}$ \\ ${ }^{1}$ Medical College, Hunan Normal University, Changsha 410006, China \\ ${ }^{2}$ School of Pharmaceutical Sciences, Central South University, Changsha 410013, China \\ Correspondence should be addressed to Peng Yu; peng.yu@csu.edu.cn
}

Received 3 June 2014; Revised 17 September 2014; Accepted 17 September 2014; Published 29 September 2014

Academic Editor: Toyohide Takeuchi

Copyright (c) 2014 Lingli Mu et al. This is an open access article distributed under the Creative Commons Attribution License, which permits unrestricted use, distribution, and reproduction in any medium, provided the original work is properly cited.

\begin{abstract}
Strong acidic drugs are a class of chemical compounds that normally have high hydrophilicity and large negative charges, such as organophosphatic compounds and organosulphonic compounds. This review focuses on sample preparation and separation methods for this group of compounds in biological matrices in recent years. A wide range of separation techniques, especially chromatographic method, are presented and critically discussed, which include liquid chromatography (e.g., ion-pair and ionexchange chromatography), capillary electrophoresis (CE), and other types. Due to the extremely low concentration level of target analytes as well as the complexity of biological matrices, sample pretreatment methods, such as dilute and shoot methods, protein precipitation (PP), liquid-liquid extraction (LLE), solid-phase extraction (SPE), degradation, and derivatization strategy, also play important roles for the development of successful analytical methods and thus are also discussed.
\end{abstract}

\section{Introduction}

The main members of strong acidic drugs include organophosphatic compounds and organosulphonic compounds, which have emerged as promising candidates for treating a wide range of diseases such as AIDS [1], cancer [2], osteoarthritis [3], Alzheimer's disease, and cardiovascular disorders [4]. For example, nucleoside reverse transcriptase inhibitors (NRTI), the first safe and effective agents for the treatment of patients infected with HIV, must be selectively phosphorylated to their active triphosphate moieties (NRTITP) within human peripheral blood mononuclear cells [1]. Taurine, a major metabolite of sulfur-containing amino acid in mammals, plays a very important role in several essential biological processes, and recently existence of taurine in urine was found to be an indicator of bladder cancer [5]. Chondroitin sulfate (CS) and dermatan sulfate (DS) show important functions in central nervous system development, wound repair, infection, growth factor signaling, morphogenes and cell division, differentiation, and migration in addition to osteoarthritis and their conventional structural roles.
Furthermore, CS is used and recommended by the European League against Rheumatism (EULAR) as a SYSADOA (symptomatic slow-acting drug for osteoarthritis) drug in Europe in the treatment of knee and hand osteoarthritis [3].

Due to the high hydrophilicity, there are numbers of difficulties and challenges for analyzing these strong acidic drugs. (a) These compounds contain one or more strong acidic groups, and they are hard to be retained on common HPLC chromatographic columns like $\mathrm{C}_{18}$ or $\mathrm{C}_{8}$ columns, which makes it more difficult to separate them from hydrophilic biological interference material. (b) Many of these compounds have several $\mathrm{pKa}$ values which may span the entire $\mathrm{pH}$ range, making it more challenging to establish a chromatographic separation method with a simple isocratic mobile phase. (c) Some of these compounds have weak ultraviolet absorption or fluorescence, which makes it hard to inspect them while separating. (d) To measure these sorts of analytes at low concentration levels, sensitive quantitative methods are required especially when applied to calculate pharmacokinetic and pharmacodynamic parameters. (e) For the complexity and challenges experienced during sample preparation of these 
compounds in biological fluids and tissues, special techniques are required to extract the target analytes from biological matrices, to separate them from interfering materials, and then to quantify and characterize them. On related topics, the separation methods for bisphosphonate active pharmaceutical ingredients in pharmaceuticals and biological material were reviewed in 2008 by Zacharis and Tzanavaras [6], and chromatographic separation methods for the determination of therapeutic oligonucleotides were reviewed by McGinnis et al. in 2012 [4].

Trying to find proper separation methods to resolve the accurate and precise quantification of strong acidic drugs in complex biological matrix samples, we searched and analyzed the related information in dozens of academic articles ranging from 2002 to date. In this review we focus on bioanalytical methods (especially chromatographic methods) for analyzing strong acidic compounds, mainly organophosphatic compounds and organosulphonic compounds. The review covers analytical methods using liquid chromatography (e.g., ion-pair, ion-exchange, and hydrophilic interaction chromatography), capillary electrophoresis (CE), and other analytical methods. Due to the extremely low concentration levels of target analytes as well as the complexity of biological matrices, sample pretreatment methods such as dilute and shoot method, protein precipitation (PP), liquid-liquid extraction (LLE), solid-phase extraction (SPE), degradation, and derivatization strategy are also explored.

\section{Sample Preparation}

Generally, biological samples, such as urine, serum or plasma, and tissues, should not be directly injected into a separation system due to matrix complexity. To resolve the problem, a sequence of sample treatment steps is performed prior to analysis. The main purposes of sample pretreatment are to remove unwanted interfering substances, suppress matrix effects and enhance selectivity, convert the analytes to a compatible form for analysis, and if possible increase sensitivity through preconcentration [6].

Dilute and shoot method is one of the simplest approaches for sample preparation. Using this method, a fast, sensitive, and noninvasive LC-MS/MS technique was developed and validated to separate and quantitatively determine taurine and related biomarkers in urine matrices by Gamagedara et al. [5]. This method is simpler and more time-saving than other sample preparation methods, and it is much suitable for the biological fluids like urine which contains relatively high concentration levels of target analytes as well as a small quantity of proteins and other endogenous substances. But dilute and shoot method is hard to apply to other biological samples such as plasma and serum.

Protein precipitation is a simple procedure to remove proteins from plasma and other biological matrices. Briefly, the reagents that are used to precipitate proteins from biological samples mostly include inorganic acids, organic acids, and organic solvents, such as perchloric acid (PCA) $[7,8]$, trichloroacetic acid (TCA) [9], acetonitrile (ACN) [10-15], and methanol [2, 16, 17]. After adding protein precipitating reagent, the mixture is usually needed to be centrifuged to remove the denatured protein [18]. Using trichloroacetic acid as protein precipitation reagent, Losa et al. isolated gemcitabine di- and triphosphate in peripheral blood mononuclear cells (PBMC) [9]. In this study, the temperature was controlled at $4^{\circ} \mathrm{C}$ during sample preparation to maintain the stability of nucleotide triphosphates, and after protein precipitation Freon (1,1,2-trichlorotrifluoroethane)trioctylamine $(4: 1, \mathrm{v} / \mathrm{v})$ was used to yield clean extracts and it neutralizes the samples. Protein precipitation can also attain other objectives simultaneously. For instance, sodium octanoate was used as binding competition to replace pcresyl sulfate and indoxyl sulfate from albumin followed by protein precipitation for sample preparation [19]. This method allowed direct quantification of indoxyl sulfate and p-cresyl sulfate which are protein-bound marker molecules in chronic kidney disease. Chen et al. [14] used acetonitrile to precipitate protein while extracting indocyanine green from dog plasma and bile. The high efficiency of protein precipitation has also been proved in many studies while preparing sorts of strong acidic drug samples with different biological matrices including serum $[16,19,20]$, plasma $[7,10,12,14-$ $17,21]$, cerebrospinal fluid [7], urine $[12,13,16]$, bile [20], brain tissue $[2,7,21]$, PBMC [9], and liver [10, 16]. However, the dilution effects of adding protein precipitation reagents sometimes become a problem when the concentration levels of target analytes in biological matrices are extremely low. Under these conditions, the samples are often needed to be further concentrated by using procedures such as liquidliquid extraction (LLE) and solid-phase extraction (SPE) to improve method sensitivity before sample analysis.

LLE is a simple and efficient method for the separation and concentration of relatively hydrophobic compounds. However, most of strong acidic drugs in biological fluids and tissues present in phosphorylated, sulfated, or sulfonated forms, so the strong acidic drugs are usually classified as highly hydrophilic compounds. In many cases LLE is not an effective sample preparation method for these compounds. Thus, it is nearly impossible to extract them with organic solvent from biological samples. A strategy to this problem is to add certain strong acid to adjust the $\mathrm{pH}$ value, so that their ionization is suppressed and they can be treated as hydrophobic molecules. For example, Du and Eddington [22] described a LLE method using trifluoroacetic acid as ionizationsuppressing reagent for the extraction of chondroitin sulfate disaccharides. Actually, controlling the $\mathrm{pH}$ values is not always an effective way to suppress ionization for strongly acidic compounds. Therefore, adding proper counterion to perform the ion-pair extraction becomes a better choice, in which the target analytes and the counterions are combined into ion-pair complexes which can be easily extracted with usual organic solvents. Briefly, adding a special ion-pair reagent into the sample solution allows target analytes to form ion-pair complexes with the counterions of opposite charge, and then the ion-pairs possess higher partition coefficients than the original analytes, and as a result the transferring of the target drugs into the extractant (organic) phase is enhanced. Xu et al. [23] applied an ion-pair liquid-liquid extraction method while successfully extracting nonsteroidal 
anti-inflammatory drugs from water-based samples. In this study, using tetrabutylammonium hydrogen sulfate as the ion-pair reagent, the formed ion-pairs had much higher solubility in organic phase (ethyl acetate) than the original compounds, which facilitated the extraction process. But the difficulty during selecting and optimizing the operation conditions is the main drawback of this method. To the best of our knowledge, till today only few studies applying ionpair extraction method to extract strong acidic compounds in biological matrix have been published.

Usually, one of the major challenges in developing a method for the simultaneous quantitative analysis of target compound and its metabolites is that some of the target analytes are highly water-soluble while others are highly hydrophobic. Thus, there is usually loss of some analytes using the standard two-phase LLE methods which could sacrifice the recovery and the method sensitivity for some of those target analytes. So, protein precipitation was sometimes used for the extraction of sphingosine and sphingosine 1phosphate prior to the LC-MS/MS analysis because of its simplicity [24]. However, Emotte et al. [25] found that for samples originating from blood protein precipitation was not always compatible with LC-MS/MS analysis as they observed some sensitivity loss and MS clogging during the sample analysis. Consequently, they used $\mathrm{C}_{18}$ SPE columns for the extraction of fingolimod and fingolimod-phosphate from biological matrices, and the SPE step was automated and operated online with LC-MS/MS detection, offering lower limit of detection (LOD) by sample preconcentration while saving time. Another example is that Satonin et al. [26] applied a single-step SPE method to separate and concentrate 5-hydroxy-6-methoxy duloxetine sulfate in plasma, in which the average extraction recovery of 5-hydroxy-6methoxy duloxetine sulfate in plasma was approximately $97 \%$. Huang et al. [10] described an analytical procedure for monitoring bile acids and their sulfate metabolites in mouse bile and urine using Supelclean LC-18 SPE using onestep sample preparation method which could also achieve high extraction recovery. Furthermore, SPE has been successfully used for sample pretreatment of various strong acidic compounds such as the phosphorylated metabolites of nucleoside [27, 28], sulfate metabolites of bile acids [10, 11], ATP, ADP, AMP, and guanine nucleotides [29]. Compared with the protein precipitation and LLE, SPE shows the following advantages. (a) Because the separation mechanism of SPE is based on interaction between the functional groups of analyte and packing material, the method is reproducible and easily realizes autostandardization procedure. (b) SPE can be automated and operated online with LC-MS/MS detection, thus offering lower limit of detection (LOD) by sample preconcentration while saving time.

In addition to those traditional methods for sample preparation of strong acidic drugs, some special methods are also used. Robbins et al. [27] described an anion exchange solid-phase extraction combined with degradation method for extracting triphosphate metabolites of zidovudine, lamivudine, and abacavir. This research successfully separated triphosphate metabolites of zidovudine, lamivudine, and abacavir from their mono- and diphosphates and degraded these phosphorylated metabolites to the parent drugs which were more suitable for chromatographic analysis. Huang et al. [30] reported that sulfate group losses were quite common while applying tandem mass spectrometry as detector for analyzing acidic compounds, such as glycosaminoglycans, which made the analysis of organosulphonic mixture highly problematic due to the simultaneous presence of many product ions from sulfated and nonsulfated drugs. Sufficient fragmentation information for definitive assignment of sulfation sites is not always available, even under optimized conditions, which makes certain methods (e.g., LC-MS/MS and CE-MS) more difficult for identifying sulfation position of glycosaminoglycans oligosaccharides with high degrees of heterogeneity. So they developed a chemical-derivatization strategy to convert the chondroitin sulfate oligosaccharides to compatible forms for chromatographic separation, in which chondroitin sulfate hexasaccharides were first premethylated to protect free hydroxyl and carboxyl groups, and then the sulfate groups were removed and the resulting active hydroxyl groups were acetylated. The purpose of the chemical-derivation strategy is to use a more stable and distinguishable group that can be differentiated from premethylated groups by both mass spectrum and chromatographic retention time to replace the labile sulfate groups. This strategy allows the chromatographic separation and structural identification of different sulfated glycosaminoglycans oligomers. In another study, chondroitin sulfate disaccharides in dog and horse plasma were extracted using LLE method and then digested by chondroitinase, followed by derivatization with $1 \%$ dansylhydrazine in ethanol at $40^{\circ} \mathrm{C}$ for $3 \mathrm{~h}$ and finally separation on a $\mathrm{C}_{18}$ column and detection using a fluorescence detector [22]. The degradation or derivatization methodology can transform strong hydrophilic analytes into the hydrophobic forms, thereby increasing their retention time on the reverse-phase chromatographic column, which often improves the stability as well as analytical sensitivity of analytes. But these special methods are usually laborconsuming and time-costing, which may bring inevitable errors.

\section{Reversed-Phase Liquid Chromatography}

Reversed-phase high-performance liquid chromatography (RP-HPLC) continues to dominate the chromatographic separation of chemical drugs and other small molecules, despite the availability of other HPLC modes such as ion-exchange and normal phase chromatography. Lu et al. [31] developed and validated a novel stability-indicating RP-HPLC method using two oxocyclic organic modifiers in the mobile phase, which was used to separate a total of 32 potential impurities and degradation products from betamethasone sodium phosphate and betamethasone acetate. In this study, special selectivity was achieved by the combination of two oxocyclic solvents, namely, tetrahydrofuran (THF) and 1,4-dioxane, which were used as the organic modifiers of the mobile phases. Further improvement of peak symmetry and separation 
efficiency was achieved by using two chaotropic agents (trifluoroacetic acid and potassium hexafluorophosphate) in the mobile phases. Meléndez et al. [32] developed and validated a sensitive and specific method using high-performance liquid chromatography-tandem mass spectrometry (HPLCMS/MS) for the determination of ribavirin monophosphate (RBV-MP) and ribavirin triphosphate (RBV-TP) in cells. In this method, ribavirin phosphorylated metabolites were extracted using anion exchange solid-phase extraction (SPE) prior to the simultaneous HPLC-MS/MS determination of RBV-MP and RBV-TP. Gamagedara et al. [5] systematically investigated and optimized the conditions that could affect LC separation and MS/MS detection to establish a method for separation and quantitative determination of taurine and related biomarkers in urine matrices. This method can completely separate the target compounds (using a phenylhexyl column) within $10 \mathrm{~min}$. De Loor et al. [19] employed RP-HPLC based on SunFire $C_{18}$ column with fluorescence detector (FLD) setting at $\lambda$ ex $260 \mathrm{~nm} / \lambda$ em $288 \mathrm{~nm}$ for $\mathrm{p}$ cresyl sulfate and $\lambda$ ex $280 \mathrm{~nm} / \lambda$ em $390 \mathrm{~nm}$ for indoxyl sulfate to determine indoxyl sulfate and p-cresyl sulfate in the serum samples from healthy subjects and uremic patients.

Totally, due to strong hydrophilicity the target analytes have poor retention on the chromatographic column, so in many cases common RP-HPLC method cannot successfully separate these ionized compounds and thus restricts its application in this kind of bioanalysis.

\section{Ion-Pair Liquid Chromatography}

In some cases, $\mathrm{pH}$ adjustment of the mobile phase in RPHPLC fails to separate mixtures of very hydrophilic organic compounds with ionic character, while ion-pair liquid chromatography (IPLC) is one of the most popular approaches to achieve efficient separations of such species. IPLC can be easily performed by modifying the mobile phases used in the RP-HPLC on the same analytical columns. An amphiphilic anion or cation, usually an alkyl sulphonic acid or salt and alkyl quaternary amine, respectively, is added to the mobile phases to enhance the retention of analytes bearing opposite charges. Briefly, tetrabutylammonium salt $[9,20,29$, 33-36], hexylamine [37-39], dimethylhexylamine (DMHA) [25], and tributylamine [40] were usually used as ion-pair reagents for measuring strong acidic compounds in IPLC methodology. With adequate selectivity, this method allows universal UV detection for quantitative analysis and is more easily interfaced with mass spectrometry to provide exact mass data and MS/MS characterization.

Lefebvre et al. [34] reported a simple, rapid, and reproducible analytical method for the simultaneous quantification of zidovudine (AZT) and its monophosphate (AZT-MP) in cell extracts by IPLC. Due to the relatively low octanolwater partition coefficients (log P) of AZT-MP, it is quite challenging to establish a chromatographic method because this highly water-soluble compound shows extremely poor retention on ordinary reversed-phase columns. Thus, a RPHPLC approach with addition of triethylammonium acetate as ion-pairing reagent to the mobile phase was developed to improve the chromatographic selectivity of AZT, AZTMP, and the internal standard on an analytical RP-HPLC column. Calaf et al. [20] developed a rapid, simple, and very sensitive ion-pair liquid chromatography method to quantify several uremic solutes in a unique sample of biological fluid: phenol, indole-3-acetic acid, p-cresol, indoxyl sulfate, and $\mathrm{p}$-cresol sulfate. The chromatographic separation was successfully obtained by using tetrabutylammonium iodide as ion-pair reagent. Moreover, in this study an isocratic flow was employed instead of gradient flow, which avoided time-consuming column reequilibrium. Emotte et al. [25] established a reliable method for the simultaneous determination of fingolimod (FTY720) and its active metabolite FTY720-phosphate (FTY720-P) in human blood sample, in which the mobile phases consisting of dimethylhexylamine (DMHA) solution and acetonitrile-isopropanol (80/20, v/v) were optimized for the separation of fingolimod phosphate and fingolimod from the interfering materials. The chromatographic separation condition showed that ion-pair reagents with more volatility such as DMHA could also be successfully employed to retain ionizable compounds on analytical column. Xie et al. [41] presented an IPLC method using evaporative light-scattering detection (ELSD) for identification and simultaneous determination of four bisphosphonates (alendronate, pamidronate, zoledronic acid, and etidronate) which were not retained on hydrophobic column and lack chromophore for detection. In this study, organic amines including n-butylamine, n-hexylamine, and $\mathrm{n}$-octylamine were investigated as ion-pair reagents for separation of the four compounds and were evaluated along with n-amylamine in order to find an appropriate retention of the four compounds. And, as a result, n-amylamine was selected as the most suitable volatile additive agent. This newly developed method sufficiently separated analytes from each other on a Phenomenex $\mathrm{C}_{18}$ column and enabled direct analysis of bisphosphonates without any derivatization.

IPLC has been widely used to selectively separate ionizable and ionic organic compounds in various samples. However, this technique is generally not compatible with ESIMS because of the use of nonvolatile mobile phase solvents, buffers, or salts. Even given the availability of some relatively volatile ion-pairing reagents, ion suppression and other issues related to method robustness are still insolvable problems with LC-MS analysis. Researches on new ion-pair agents are mainly focused on ensuring that the ion-pairing buffer is compatible with electrospray ionization-mass spectrometry.

\section{Ion-Exchange Liquid Chromatography}

Ion-exchange liquid chromatography is a separation technique used to analyze anions and cations in solution. Ionexchange chromatography with UV and other detectors is an excellent method for separating charged molecules, which means it is also amenable to multiply charged strong acidic compounds. Motoyama et al. [42] reported an online multidimensional LC method using an anion and cation exchange (ACE) mixed bed for the first separation of peptides and phosphopeptides. The mixed-bed ion-exchange 
resin improved peptide recovery over strong cation-exchange (SCX) resins alone and showed better orthogonality than reversed-phase separations in two-dimensional separations. The application of this method to phosphopeptide-enriched samples increased phosphopeptide identifications by $94 \%$ over SCX alone. Unlike previous methods that used anion exchange to change selectivity or enrich phosphopeptides, the proposed format was unique for it works with typical acidic buffer systems which could be used with electrospray ionization, making it feasible for online multidimensional LC-MS/MS applications. In this study, the proposed anion and cation exchange mixed-bed system was effective, simple to implement, and useful for a variety of analyses including online chromatographic enrichment of acidic phosphopeptides. Liu et al. [43] developed and validated a rapid, direct, and stability-indicating method for analysis of etidronate, a bisphosphonate compound without UV chromophore, in which a mixed-mode column was used to separate etidronate from its impurities in an $8 \mathrm{~min}$ gradient method and a charged aerosol detector (CAD) was used for detection. In this study, various columns with strong ion-exchange characteristics were explored and the mixed-mode columns provided satisfactory separation and retention for all peaks of interest. Etidronate exhibited four pKa values (1.35, 2.87, 7.03, and 11.3) that spanned the entire $\mathrm{pH}$ range, which resulted in multiple charged ions in solution and caused poor peak shape. The peak shapes were significantly improved when the new mixed-mode anion-exchange reversed-phase column, Primesep SB, was used. This might be attributed to the fact that the Primesep SB packing particles were bound using a bulky basic group with a pKa value around 13, and there was more space between ligands. Compared with other published methods for separation of etidronate [41, 44], this method demonstrated sufficient sensitivity under the condition that neither ion-pair reagents nor time-consuming derivatization operation was used.

The capability of ion-exchange liquid chromatography to separate certain ionized analytes from other hydrophilic molecules in biological matrices, as well as its high tolerance for salts, significantly simplifies the extraction of biological samples. These unique advantages confer on ion-exchange chromatography an indispensable role in the pharmacokinetic and pharmacodynamic studies of strong acidic drugs. But this technique has the same problem as that of ionpair liquid chromatography; that is to say, it is generally not compatible with ESI-MS because of the use of nonvolatile mobile phase solvents, buffers, or salts.

\section{Hydrophilic Interaction Liquid Chromatography}

Hydrophilic interaction chromatography (HILIC) may be an interesting alternative to reversed-phase liquid chromatography for the separation of strong hydrophilic compounds. The HILIC mode was introduced by Alpert in 1990 [45] and later used in tandem with ESI-MS to separate and characterize hydrophilic low-molecular-weight compounds such as amino acids, peptides, glycoconjugates, and organic acids without derivatization [46, 47]. In the HILIC mode, an aqueous-organic mobile phase (containing high proportion of organic solvent) is used with a hydrophilic stationary phase to provide seemingly normal-phase retention behavior $[45,48]$, and hydrophilic compounds are retained longer than hydrophobic ones and the hydrophilic mobile phase component (usually water) is the stronger solvent.

Goutier et al. [49] developed and validated a method for the determination of cAMP, ATP (adenosine triphosphate), and other nucleotides in a biological system by combining zwitterionic hydrophilic interaction liquid chromatography and tandem mass spectrometry (MS/MS). Because of the extreme hydrophilicity of nucleotides, especially the presence of (multiple) phosphate groups, most LC methods use anion-exchange chromatography or reversed stationary phases combined with ion-pair agents. Unfortunately, both of the two methods are not compatible to be used in combination with MS/MS analysis. Instead, hydrophilic interaction liquid chromatography is able to separate very hydrophilic compounds without the addition of ion-pair agents or other nonvolatile modifiers into the mobile phase, which makes it compatible with mass spectrometry analysis. Most of the biologically active cyclic nucleotides can be used as zwitterions, for their purine base and phosphate groups, and potentially be analyzed in positive or negative electrospray ionization mode. De Person et al. [50] developed and validated a procedure based on hydrophilic interaction chromatography coupled to tandem mass spectrometry for the simultaneous determination of underivatized taurine and methionine in fluid samples. Satisfactory separation was obtained on an Astecap Hera $\mathrm{NH}_{2}$ column $(150 \mathrm{~mm}$ $\times 4.6 \mathrm{~mm}, 5 \mu \mathrm{m})$ with methanol-water $(60: 40, \mathrm{v} / \mathrm{v})$ as the mobile phase. This method could be applied to other types of biological samples such as plasma or urine. In another study, a sensitive liquid chromatography tandem mass spectrometry (LC-MS/MS) method based on a Waters Atlantis HILIC silica column equipped with a Phenomenex $\mathrm{C}_{18}$ (ODS) column was developed to simultaneously quantify amino acids and myoinositol (e.g., taurine and phosphocholine) in mouse brain [2]. A HPLC method based on HILIC was developed by Mora et al. [51] for the simultaneous analysis of adenosine triphosphate (ATP), adenosine diphosphate (ADP), adenosine monophosphate (AMP), inosine monophosphate (IMP), inosine (Ino), hypoxanthine ( $\mathrm{Hx}$ ), and nicotinamide adenine dinucleotide $(\mathrm{NAD}+)$ in meat samples. A comparison between the concentrations of the compounds measured by HILIC using a ZIC-pHILIC column and by IP-RP-HPLC using a Zorbax Eclipse $\mathrm{XDB}-\mathrm{C}_{18}$ column was also made, in which very good agreement between the two sets of data was obtained. Thus, being a valid and reliable method to analyze ATP and its metabolites, HILIC can be considered as an interesting alternative to other methodologies.

\section{Capillary Electrophoresis}

The versatility and a variety of modes of capillary electrophoresis (CE) imply that almost all molecules and even whole organisms can be separated using sorts of powerful 
CE modes such as capillary zone electrophoresis (CZE), isotachophoresis, micellar electrokinetic chromatography (MEKC), isoelectric focusing, and capillary electrochromatography (CEC). This makes CE quite useful in situations where other liquid phase separation techniques are limited or impractical. The main advantages of capillary electrophoretic techniques include high separation efficiencies, low sample consumption, short analysis time, low reagents consumption, applicable automation, and low volumes of waste $[52,53]$.

Metabolite identification and metabolite profiling are of major importance in the pharmaceutical and clinical researches. However, highly hydrophilic and ionic substances are rarely included in most of these researches only because of the lack of applicable analytical methods. Bunz et al. [54] presented a method for the determination of urinary sulfates, sulfonates, phosphates, and other anions of strong acids. The method comprised a CE separation using an acidic butyl glycidyl ether (BGE) solution $(\mathrm{pH} \leq 2)$ and anodic detection by MS via negative ESI. In this way, sulfates and sulfonates were detected in the first part of the electropherogram, followed by phosphates and potentially highly acidic carboxylates. This method could be complementary to the previous existing methods for metabolite characterization in urine. Zinellu et al. [55] reported an ultrafast method to detect adenosine $5^{\prime}$-triphosphate, adenosine $5^{\prime}$-diphosphate, and adenosine $5^{\prime}$-monophosphate in red blood cells using pressure-assisted capillary electrophoresis. $20 \mu \mathrm{mmol} \cdot \mathrm{L}^{-1}$ sodium acetate buffer at $\mathrm{pH} 3.80$ was used as running electrolyte, and the separation was performed using the condition of a CE voltage of $25 \mathrm{kV}$ and an overimposed pressure of 0.2 psi from inlet to outlet. In 2007-2008, the adulteration of raw heparin with oversulfated chondroitin sulfate (OSCS) produced a global crisis, which resulted in extensive revisions to the pharmacopeia monographs and prompted the FDA to recommend the development of additional methods for the analysis of heparin purity. As a consequence, a wide variety of innovative analytical approaches were developed for the quality assurance and purity analysis of unfractionated and low-molecular-weight heparins. Electrophoresis techniques were preferred for the sensitive separation, detection, and partial structural characterization of heparin contaminants. For the proposed CE method provides only partial separation of oversulfated chondroitin sulfate (OSCS) contaminant from heparin, Somsen et al. [56] developed an improved CE method that was especially useful for the reliable quantification of OSCS and dermatan sulfate (DS) impurities in heparin. In this study, parameters, such as type and concentration of background electrolyte, capillary temperature, sample concentration, and injection volume, were all investigated and optimized. Using high concentrations of Tris phosphate ( $\mathrm{pH}$ 3.0) as background electrolyte in combination with a $25 \mu \mathrm{m}$ internal diameter (ID) capillary, the presented method provided good separations of OSCS and DS from heparin without causing excessive currents and Joule heating. Because of the possibility of injecting relatively large sample volumes and high concentrations of heparin, OSCS could be detected down to the $0.1 \%$ level, despite the weak UV absorbance of these sulfated glycosaminoglycans. Loegel et al. [57] developed a CE method for the separation of heparin, dermatan sulfate, chondroitin sulfate (or hyaluronan), and the impurity of OSCS using polyamine-containing electrolyte, ethylenediamine (EDA). It was possibly one of the first CE methods which successfully made use of a $50 \mu \mathrm{m}$ ID fused silica capillary for analysis of OSCS impurity in heparin samples. Using the design optimization software, the $\mathrm{CE}$ run buffer containing $200 \mathrm{mM}$ EDA and $45.5 \mathrm{mM}$ phosphate was the best for peak resolution. The migration time of OSCS was the longest instead of shortest while compared to previous methods using Tris or lithium phosphate containing electrolytes.

A CE method for the determination of the ethanol consumption marker (ethyl sulfate, EtS) in human urine was developed and validated [58]. Analysis was performed in negative polarity mode with a background electrolyte composed of $15 \mathrm{mM}$ maleic acid, $1 \mathrm{mM}$ phthalic acid, and $0.05 \mathrm{mM}$ cetyltrimethylammonium bromide $(\mathrm{CTAB})$ at $\mathrm{pH}$ 2.5. This buffer system provided selective separation for EtS and vinylsulfonic acid (employed as internal standard) from urine matrix components. The proposed method seemingly was a convenient and valuable alternative for the comparably expensive and tedious LC-MS methods for the determination of EtS in human urine. In another study, Caslavska et al. [59] reported a confirmation analysis of ethyl glucuronide (EtG) and EtS in human serum and urine after intake of alcoholic beverages using CZE coupled to sheath liquidbased electrospray ionization (ESI) and multiple-stage ion trap mass spectrometry $\left(\mathrm{MS}^{n}\right)$. Electrophoretic separations were performed in uncoated fused-silica capillaries using a pH 9.5 ammonium acetate background electrolyte in normal polarity mode. CZE-MS and CZE-MS ${ }^{2}$ results obtained after injection of solid-phase extracts for EtG and EtS and of diluted urine confirmed the presence of EtG and EtS in samples whose concentration levels were previously determined by CZE with indirect UV detection.

Tseng et al. [13] demonstrated a simple and efficient method for the simultaneous separation and stacking of neurotransmitters in capillary electrophoresis with UV detection. By using poly diallyldimethylammonium chloride (PDDAC) as a buffer additive, the fast and reversed electroosmotic flow (EOF) was observed. Moreover, the mobility of indolamines and catecholamines decreased as the PDDAC concentration increased. Based on the difference of mobility between the presence and absence of PDDAC, the analytes were simply stacked between the boundary of the sample zone and the background electrolyte containing PDDAC. For the determination of taurine in body fluids as well as in human skin extract, da Silva et al. [60] developed simple methods using $\mathrm{CE}$, and the qualitative and quantitative comparisons between fluorescence detection after precolumn derivatization and direct detection using integrated pulse amperometry were performed.

\section{Discussion and Conclusion}

For bioanalytical researchers, it is really a difficult problem to separate and analyze strong acidic compounds in biological matrices. The existence of phosphonic, sulphonic groups in 
their chemical structures brings strongly ionic characters and remarkably increased hydrophilicity. Moreover, some members of this kind of compounds lack chromophores functional groups; thus convenient direct UV detection is often impracticable. Therefore, resolving the problems in bioanalysis of strong acidic compounds may greatly promote the pharmacokinetic and pharmacodynamic studies of this kind of drugs as well as clinical applications of these compounds. This review attempts to highlight the experiences with successful establishment and application of separation methods of strong acidic drugs and the challenges the researchers are facing.

In this review, the methods using RP-HPLC, IPLC, ionexchange LC, HILIC, CE, and so forth, for analysis of strong acidic compounds, are reviewed. Among these methods, RP-HPLC is the most powerful and popular method for the analysis of moderately hydrophilic and hydrophobic compounds, but it cannot always satisfactorily separate these highly hydrophilic compounds due to the poor retention of these analytes on common reversed-phase chromatographic columns. IPLC has been extensively used to selectively separate ionizable and ionic organic compounds in various samples. However, this technique is generally not compatible with ESI-MS because of the use of nonvolatile mobile phase solvents, buffers, or salts. In addition, even considering the availability of relatively volatile ion-pairing reagents, ion suppression and other issues related to method ruggedness are still major problems when IPLC is combined with LC-MS for the analysis of hydrophilic analytes in biological matrices. Ion-exchange chromatography is an excellent method for separating charged molecules and, accordingly, is amenable to large multiple charged strongly hydrophilic compounds. The unique advantages of ion-exchange chromatography (e.g., high tolerance for salts and significantly simplified sample extractions) make it an indispensable role in the bioanalysis of strong acidic compounds. But this technique has the same problem as that of ion-pair liquid chromatography; namely, it is generally not compatible with ESI-MS. Hydrophilic interaction chromatography is able to separate very hydrophilic compounds without the addition of ionpairing agents, and it can use mobile phases which are compatible with ESI-MS. It may be an interesting and efficient alternative technique to other methods (e.g., ion-pair and ion-exchange chromatography) for the separation of strongly hydrophilic compounds. $\mathrm{CE}$ is an efficient technique for the separation and quantification of both ionic and neutral species, but the technology of interface between CE and MS still needs to be improved. Furthermore, the high concentrations of salt in the electrolytes used in CE often lead to the loss of ESI-MS detection sensitivity.

On the other hand, sample preparation is of key importance as it influences both selectivity via effective matrix removal and sensitivity via preconcentration. Dilute and shoot method is a simpler and time-saving approach for sample preparation. But it is only suitable for the biological fluids (e.g., urine) which contain low concentrations of proteins and high concentrations of target analytes, and it cannot apply to most biological samples such as plasma and serum. In addition, the direct dilute and shoot method may lead to poor separation and detection sensitivity of the analytes. Protein precipitation is a widely accepted method for pretreatment of strong acidic compounds-containing biological samples prior to liquid chromatographic analysis. This method has been proven quite effective, but the solution obtained after pretreatment often needs to be further concentrated using other procedures such as LLE or SPE. Liquidliquid extraction is also a simple and efficient method for the separation and concentration of relatively hydrophobic compounds. However, for highly hydrophilic compounds such as the strong acidic compounds, LLE is often not an effective extraction procedure. A strategy to the problem is to use certain strong acid to modify the $\mathrm{pH}$ of the sample matrix or to add ion-pair reagents. Alternative approaches include SPE or combination of SPE and protein precipitation when higher selectivity is required. Compared with protein precipitation and LLE, SPE is more reproducible, easier to transfer between the laboratories, and more suitable for procedure standardization and automation. Additionally, this method has better selectivity and enrichment capability, higher precision and accuracy, and other prominent advantages. For some highly hydrophilic compounds with poor stability or without chromophores, they must be firstly degraded and/or derivatized and then separated and quantified. The degradation or derivatization methodology can transform highly hydrophilic analytes into the moderately hydrophilic or hydrophobic forms, thereby prolonging their retention time on the chromatographic column and improving the stability and analytical sensitivity of analytes. But these special methods are generally labor-consuming and timecosting, and the more complex sample procedures may bring some inevitable errors.

Although LC-MS and LC-MS/MS nowadays have the dominating role in bioanalysis, applications of these techniques to the determination of highly hydrophilic compounds are still limited; there are especially so many unsolved problems in the field of separation and determination of strong acidic drugs in biological samples. In our opinion, the coming research needs to focus on creating some novel, original, and pioneering theories which can bring completely different separation modes. The second best way is to develop more salt-tolerant and sensitive detection methods, for example, making ion-pair and ion-exchange chromatography compatible with mass spectrometry. Since sample preparation plays an indispensable role in the bioanalysis of strong acidic drugs, finding simpler and more effective sample extraction procedures should not be ignored in the future.

\section{Conflict of Interests}

The authors declare that there is no conflict of interests regarding the publication of this paper.

\section{Acknowledgments}

This work was supported by the National Natural Science Foundation of China (no. 81102499) and Hunan Science and Technology Project (no. 2011SK3261). 


\section{References}

[1] T. King, L. Bushman, P. L. Anderson, T. Delahunty, M. Ray, and C. V. Fletcher, "Quantitation of zidovudine triphosphate concentrations from human peripheral blood mononuclear cells by anion exchange solid phase extraction and liquid chromatography-tandem mass spectroscopy; an indirect quantitation methodology," Journal of Chromatography B: Analytical Technologies in the Biomedical and Life Sciences, vol. 831, no. 1-2, pp. 248-257, 2006.

[2] S. P. Bathena, J. Huang, A. A. Epstein, H. E. Gendelman, M. D. Boska, and Y. Alnouti, "Rapid and reliable quantitation of amino acids and myo-inositol in mouse brain by high performance liquid chromatography and tandem mass spectrometry," Journal of Chromatography B: Analytical Technologies in the Biomedical and Life Sciences, vol. 893-894, pp. 15-20, 2012.

[3] N. Volpi, "High-performance liquid chromatography and online mass spectrometry detection for the analysis of chondroitin sulfates/hyaluronan disaccharides derivatized with 2aminoacridone," Analytical Biochemistry, vol. 397, no. 1, pp. 1223, 2010.

[4] A. C. McGinnis, B. Chen, and M. G. Bartlett, "Chromatographic methods for the determination of therapeutic oligonucleotides," Journal of Chromatography B: Analytical Technologies in the Biomedical and Life Sciences, vol. 883-884, pp. 76-94, 2012.

[5] S. Gamagedara, H. Shi, and Y. Ma, "Quantitative determination of taurine and related biomarkers in urine by liquid chromatography-tandem mass spectrometry," Analytical and Bioanalytical Chemistry, vol. 402, no. 2, pp. 763-770, 2012.

[6] C. K. Zacharis and P. D. Tzanavaras, "Determination of bisphosphonate active pharmaceutical ingredients in pharmaceuticals and biological material: a review of analytical methods," Journal of Pharmaceutical and Biomedical Analysis, vol. 48, no. 3, pp. 483-496, 2008.

[7] P. Oeckl and B. Ferger, "Simultaneous LC-MS/MS analysis of the biomarkers cAMP and cGMP in plasma, CSF and brain tissue," Journal of Neuroscience Methods, vol. 203, no. 2, pp. 338-343, 2012.

[8] E. J. C. M. Coolen, I. C. W. Arts, E. L. R. Swennen, A. Bast, M. A. C. Stuart, and P. C. Dagnelie, "Simultaneous determination of adenosine triphosphate and its metabolites in human whole blood by RP-HPLC and UV-detection," Journal of Chromatography B: Analytical Technologies in the Biomedical and Life Sciences, vol. 864, no. 1-2, pp. 43-51, 2008.

[9] R. Losa, M. I. Sierra, M. O. Gión, E. Esteban, and J. M. Buesa, "Simultaneous determination of gemcitabine di- and triphosphate in human blood mononuclear and cancer cells by RP-HPLC and UV detection," Journal of Chromatography B: Analytical Technologies in the Biomedical and Life Sciences, vol. 840, no. 1, pp. 44-49, 2006.

[10] J. Huang, S. P. R. Bathena, I. L. Csanaky, and Y. Alnouti, "Simultaneous characterization of bile acids and their sulfate metabolites in mouse liver, plasma, bile, and urine using LCMS/MS," Journal of Pharmaceutical and Biomedical Analysis, vol. 55, no. 5, pp. 1111-1119, 2011.

[11] Y. Alnouti, I. L. Csanaky, and C. D. Klaassen, "Quantitativeprofiling of bile acids and their conjugates in mouse liver, bile, plasma, and urine using LC-MS/MS," Journal of Chromatography B, vol. 873, no. 2, pp. 209-217, 2008.

[12] A. Liu, Y. Chen, Z. Yang et al., "New metabolites of fenofibrate in Sprague-Dawley rats by UPLC-ESI-QTOF-MS-based metabolomics coupled with LC-MS/MS," Xenobiotica, vol. 39, no. 4, pp. 345-354, 2009.
[13] W.-L. Tseng, S.-M. Chen, C.-Y. Hsu, and M.-M. Hsieh, "On-line concentration and separation of indolamines, catecholamines, and metanephrines in capillary electrophoresis using high concentration of poly(diallyldimethylammonium chloride)," Analytica Chimica Acta, vol. 613, no. 1, pp. 108-115, 2008.

[14] C. Y. Chen, R. M. Fancher, Q. Ruan, P. Marathe, A. D. Rodrigues, and Z. Yang, "A liquid chromatography tandem mass spectrometry method for the quantification of indocyanine green in dog plasma and bile," Journal of Pharmaceutical and Biomedical Analysis, vol. 47, no. 2, pp. 351-359, 2008.

[15] G. Corona, C. Elia, B. Casetta et al., "Liquid chromatography tandem mass spectrometry assay for fast and sensitive quantification of estrone-sulfate," Clinica Chimica Acta, vol. 411, no. 7-8, pp. 574-580, 2010.

[16] I. Bobeldijk, M. Hekman, J. de Vries-van der Weij et al., "Quantitative profiling of bile acids in biofluids and tissues based on accurate mass high resolution LC-FT-MS: compound class targeting in a metabolomics workflow," Journal of Chromatography B: Analytical Technologies in the Biomedical and Life Sciences, vol. 871, no. 2, pp. 306-313, 2008.

[17] S. J. Mao, S. X. Hou, Z. Liang et al., "Ion-pair reversed-phase HPLC: assay validation of sodium tanshinone IIA sulfonate in mouse plasma," Journal of Chromatography B: Analytical Technologies in the Biomedical and Life Sciences, vol. 831, no. 1-2, pp. 163-168, 2006.

[18] J. Lai, J. Wang, and Z. Cai, "Nucleoside reverse transcriptase inhibitors and their phosphorylated metabolites in human immunodeficiency virus-infected human matrices," Journal of Chromatography B, vol. 868, no. 1-2, pp. 1-12, 2008.

[19] H. de Loor, B. K. I. Meijers, T. W. Meyer et al., "Sodium octanoate to reverse indoxyl sulfate and $p$-cresyl sulfate albumin binding in uremic and normal serum during sample preparation followed by fluorescence liquid chromatography," Journal of Chromatography A, vol. 1216, no. 22, pp. 4684-4688, 2009.

[20] R. Calaf, C. Cerini, C. Génovésio et al., "Determination of uremic solutes in biological fluids of chronic kidney disease patients by HPLC assay," Journal of Chromatography B: Analytical Technologies in the Biomedical and Life Sciences, vol. 879, no. 23, pp. 2281-2286, 2011.

[21] J. R. Zgoda-Pols, S. Chowdhury, M. Wirth, M. V. Milburn, D. C. Alexander, and K. B. Alton, "Metabolomics analysis reveals elevation of 3-indoxyl sulfate in plasma and brain during chemically-induced acute kidney injury in mice: investigation of nicotinic acid receptor agonists," Toxicology and Applied Pharmacology, vol. 255, no. 1, pp. 48-56, 2011.

[22] J. Du and N. Eddington, "Determination of the chondroitin sulfate disaccharides in dog and horse plasma by HPLC using chondroitinase digestion, precolumn derivatization, and fluorescence detection," Analytical Biochemistry, vol. 306, no. 2, pp. 252-258, 2002.

[23] L. Xu, M. Jiang, and G. Li, "Injection port derivatization following sonication-assisted ion-pair liquid-liquid extraction of nonsteroidal anti-inflammatory drugs," Analytica Chimica Acta, vol. 666, no. 1-2, pp. 45-50, 2010.

[24] T. Lan, H. Bi, W. Liu, X. Xie, S. Xu, and H. Huang, "Simultaneous determination of sphingosine and sphingosine 1-phosphate in biological samples by liquid chromatography-tandem mass spectrometry," Journal of Chromatography B: Analytical Technologies in the Biomedical and Life Sciences, vol. 879, no. 7-8, pp. 520-526, 2011.

[25] C. Emotte, F. Deglave, O. Heudi, F. Picard, and O. Kretz, "Fast simultaneous quantitative analysis of FTY720 and its metabolite 
FTY720-P in human blood by on-line solid phase extraction coupled with liquid chromatography-tandem mass spectrometry," Journal of Pharmaceutical and Biomedical Analysis, vol. 58, no. 1, pp. 102-112, 2012.

[26] D. K. Satonin, J. D. McCulloch, F. Kuo, and M. P. Knadler, "Development and validation of a liquid chromatographytandem mass spectrometric method for the determination of the major metabolites of duloxetine in human plasma," Journal of Chromatography B, vol. 852, no. 1-2, pp. 582-589, 2007.

[27] B. L. Robbins, P. A. Poston, E. F. Neal, C. Slaughter, and J. H. Rodman, "Simultaneous measurement of intracellular triphosphate metabolites of zidovudine, lamivudine and abacavir (carbovir) in human peripheral blood mononuclear cells by combined anion exchange solid phase extraction and LCMS/MS," Journal of Chromatography B: Analytical Technologies in the Biomedical and Life Sciences, vol. 850, no. 1-2, pp. 310-317, 2007.

[28] L. R. Bushman, J. J. Kiser, J. E. Rower et al., "Determination of nucleoside analog mono-, di-, and tri-phosphates in cellular matrix by solid phase extraction and ultra-sensitive LC-MS/MS detection," Journal of Pharmaceutical and Biomedical Analysis, vol. 56, no. 2, pp. 390-401, 2011.

[29] P. Yeung, L. Ding, and W. L. Casley, "HPLC assay with UV detection for determination of RBC purine nucleotide concentrations and application for biomarker study in vivo," Journal of Pharmaceutical and Biomedical Analysis, vol. 47, no. 2, pp. 377$382,2008$.

[30] R. Huang, V. H. Pomin, and J. S. Sharp, "LC-MSn analysis of isomeric chondroitin sulfate oligosaccharides using a chemical derivatization strategy," Journal of the American Society for Mass Spectrometry, vol. 22, no. 9, pp. 1577-1587, 2011.

[31] J. Lu, Y. Wei, and A. M. Rustum, "A stability-indicating reversedphase high performance liquid chromatography method for simultaneous assay of two corticosteroids and estimation of their related compounds in a pharmaceutical injectable formulation," Journal of Chromatography A, vol. 1217, no. 44, pp. 69326941, 2010.

[32] M. Meléndez, O. Rosario, B. Zayas, and J. F. Rodríguez, "HPLCMS/MS method for the intracellular determination of ribavirin monophosphate and ribavirin triphosphate in CEMss cells," Journal of Pharmaceutical and Biomedical Analysis, vol. 49, no. 5, pp. 1233-1240, 2009.

[33] S. zur Nedden, R. Eason, A. S. Doney, and B. G. Frenguelli, "An ion-pair reversed-phase HPLC method for determination of fresh tissue adenine nucleotides avoiding freeze-thaw degradation of ATP," Analytical Biochemistry, vol. 388, no. 1, pp. 108114, 2009.

[34] I. Lefebvre, J.-Y. Puy, C. Perrin, and C. Périgaud, “Quantification of zidovudine and its monophosphate in cell extracts by online solid-phase extraction coupled to liquid chromatography," Journal of Chromatography B: Analytical Technologies in the Biomedical and Life Sciences, vol. 858, no. 1-2, pp. 2-7, 2007.

[35] D. P. Bhatt, X. Chen, J. D. Geiger, and T. A. Rosenberger, "A sensitive HPLC-based method to quantify adenine nucleotides in primary astrocyte cell cultures," Journal of Chromatography B: Analytical Technologies in the Biomedical and Life Sciences, vol. 889-890, pp. 110-115, 2012.

[36] M. G. Volonté, G. Yuln, P. Quiroga, and A. E. Consolini, "Development of an HPLC method for determination of metabolic compounds in myocardial tissue," Journal of Pharmaceutical and Biomedical Analysis, vol. 35, no. 3, pp. 647-653, 2004.
[37] A. M. Brustkern, L. F. Buhse, M. Nasr, A. Al-Hakim, and D. A. Keire, "Characterization of currently marketed heparin products: reversed-phase ion-pairing liquid chromatography mass spectrometry of heparin digests," Analytical Chemistry, vol. 82, no. 23, pp. 9865-9870, 2010.

[38] L. Coulier, H. Gerritsen, J. J. A. van Kampen et al., "Comprehensive analysis of the intracellular metabolism of antiretroviral nucleosides and nucleotides using liquid chromatographytandem mass spectrometry and method improvement by using ultra performance liquid chromatography," Journal of Chromatography B: Analytical Technologies in the Biomedical and Life Sciences, vol. 879, no. 26, pp. 2772-2782, 2011.

[39] L. Coulier, R. Bas, S. Jespersen, E. Verheij, M. J. van der Werf, and T. Hankemeier, "Simultaneous quantitative analysis of metabolites using ion-pair liquid chromatography-electrospray ionization mass spectrometry," Analytical Chemistry, vol. 78, no. 18, pp. 6573-6582, 2006.

[40] H. Kojima, M. Inagaki, T. Tomita, T. Watanabe, and S. Uchida, "Improved separation and characterization of lipopolysaccharide related compounds by reverse phase ion pairingHPLC/electrospray ionization-quadrupole-mass spectrometry (RPIP-HPLC/ESI-Q-MS)," Journal of Chromatography B: Analytical Technologies in the Biomedical and Life Sciences, vol. 878, no. 3-4, pp. 442-448, 2010.

[41] Z. Xie, Y. Jiang, and D.-Q. Zhang, "Simple analysis of four bisphosphonates simultaneously by reverse phase liquid chromatography using n-amylamine as volatile ion-pairing agent," Journal of Chromatography A, vol. 1104, no. 1-2, pp. 173-178, 2006.

[42] A. Motoyama, T. Xu, C. I. Ruse, J. A. Wohlschlegel, and J. R. Yates III, "Anion and cation mixed-bed ion exchange for enhanced multidimensional separations of peptides and phosphopeptides," Analytical Chemistry, vol. 79, no. 10, pp. 3623-3634, 2007.

[43] X.-K. Liu, J. B. Fang, N. Cauchon, and P. Zhou, "Direct stabilityindicating method development and validation for analysis of etidronate disodium using a mixed-mode column and charged aerosol detector," Journal of Pharmaceutical and Biomedical Analysis, vol. 46, no. 4, pp. 639-644, 2008.

[44] A. Ismail, S. Aldous, E. J. Triggs, B. A. Smithurst, and H. D. Barry, "Gas chromatographic analysis of Didronel tablets," Journal of Chromatography A, vol. 404, pp. 372-377, 1987.

[45] A. J. Alpert, "Hydrophilic-interaction chromatography for the separation of peptides, nucleic acids and other polar compounds," Journal of Chromatography, vol. 499, pp. 177-196, 1990.

[46] M. A. Strege, "Hydrophilic interaction chromatographyelectrospray mass spectrometry analysis of polar compounds for natural product drug discovery," Analytical Chemistry, vol. 70, no. 13, pp. 2439-2445, 1998.

[47] H. Schlichtherle-Cerny, M. Affolter, and C. Cerny, "Hydrophilic interaction liquid chromatography coupled to electrospray mass spectrometry of small polar compounds in food analysis," Analytical Chemistry, vol. 75, no. 10, pp. 2349-2354, 2003.

[48] T. Yoshida, "Peptide separation by hydrophilic-interaction chromatography: a review," Journal of Biochemical and Biophysical Methods, vol. 60, no. 3, pp. 265-280, 2004.

[49] W. Goutier, P. A. Spaans, M. A. W. van der Neut, A. C. McCreary, and J. H. Reinders, "Development and application of an LCMS/MS method for measuring the effect of (partial) agonists on cAMP accumulation in vitro," Journal of Neuroscience Methods, vol. 188, no. 1, pp. 24-31, 2010. 
[50] M. de Person, A. Hazotte, C. Elfakir, and M. Lafosse, "Development and validation of a hydrophilic interaction chromatography-mass spectrometry assay for taurine and methionine in matrices rich in carbohydrates," Journal of Chromatography A, vol. 1081, no. 2, pp. 174-181, 2005.

[51] L. Mora, A. S. Hernández-Cázares, M.-C. Aristoy, and F. Toldrá, "Hydrophilic interaction chromatographic determination of adenosine triphosphate and its metabolites," Food Chemistry, vol. 123, no. 4, pp. 1282-1288, 2010.

[52] K. D. Altria, A. Marsh, and C. Sänger-van de Griend, "Capillary electrophoresis for the analysis of small-molecule pharmaceuticals," Electrophoresis, vol. 27, no. 12, pp. 2263-2282, 2006.

[53] D. S. Hage, "An overview of CE in clinical analysis," Methods in Molecular Biology, vol. 919, pp. 3-10, 2013.

[54] S.-C. Bunz, W. Weinmann, and C. Neusuess, "The selective determination of sulfates, sulfonates and phosphates in urine by CE-MS," Electrophoresis, vol. 31, no. 7, pp. 1274-1281, 2010.

[55] A. Zinellu, S. Sotgia, B. Scanu et al., "Ultra-fast adenosine $5^{\prime}$-triphosphate, adenosine $5^{\prime}$-diphosphate and adenosine $5^{\prime}$ monophosphate detection by pressure-assisted capillary electrophoresis UV detection," Electrophoresis, vol. 31, no. 16, pp. 2854-2857, 2010.

[56] G. W. Somsen, Y. H. Tak, J. S. Toraño, P. M. J. M. Jongen, and G. J. de Jong, "Determination of oversulfated chondroitin sulfate and dermatan sulfate impurities in heparin by capillary electrophoresis," Journal of Chromatography A, vol. 1216, no. 18, pp. 4107-4112, 2009.

[57] T. N. Loegel, J. D. Trombley, R. T. Taylor, and N. D. Danielson, "Capillary electrophoresis of heparin and other glycosaminoglycans using a polyamine running electrolyte," Analytica Chimica Acta, vol. 753, pp. 90-96, 2012.

[58] F. A. Esteve-Turrillas, W. Bicker, M. Lämmerhofer, T. Keller, and W. Lindner, "Determination of ethyl sulfate-a marker for recent ethanol consumption-in human urine by CE with indirect UV detection," Electrophoresis, vol. 27, no. 23, pp. 47634771, 2006.

[59] J. Caslavska, B. Jung, and W. Thormann, "Confirmation analysis of ethyl glucuronide and ethyl sulfate in human serum and urine by CZE-ESI-MS ${ }^{n}$ after intake of alcoholic beverages," Electrophoresis, vol. 32, no. 13, pp. 1760-1764, 2011.

[60] D. L. P. da Silva, Y. Mrestani, H. H. Rüttinger, J. Wohlrab, and R. Neubert, "Comparison between fluorescence and pulsed integrated electrochemical detectors for the determination of taurine in human skin, urine and plasma by CE," Chromatographia, vol. 67, no. 9-10, pp. 813-817, 2008. 



\section{Carbohydrate} Chemistry

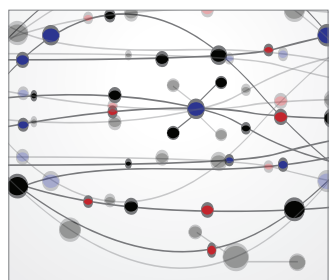

The Scientific World Journal
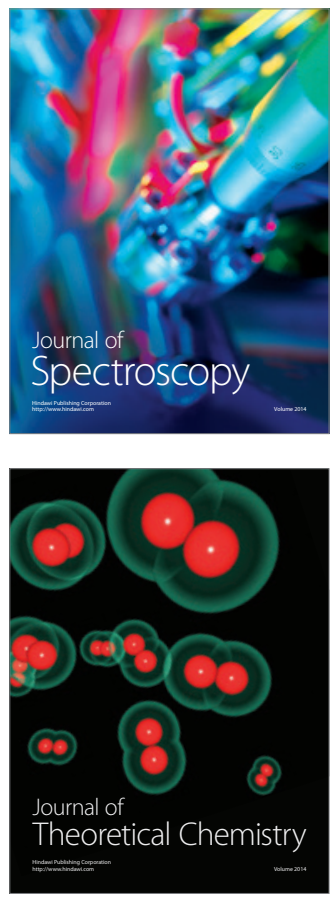


Submit your manuscripts at

http://www.hindawi.com

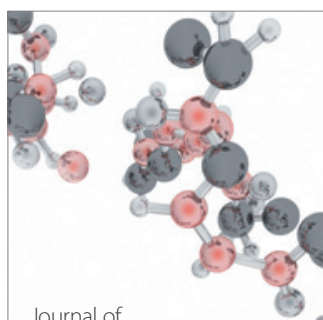

Analytical Methods

in Chemistry

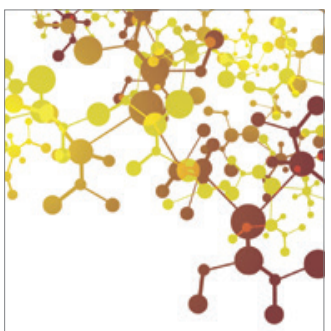

Journal of

Applied Chemistry

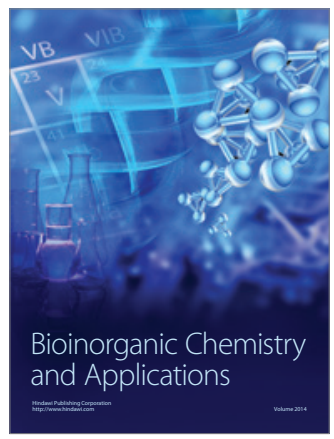

Inorganic Chemistry
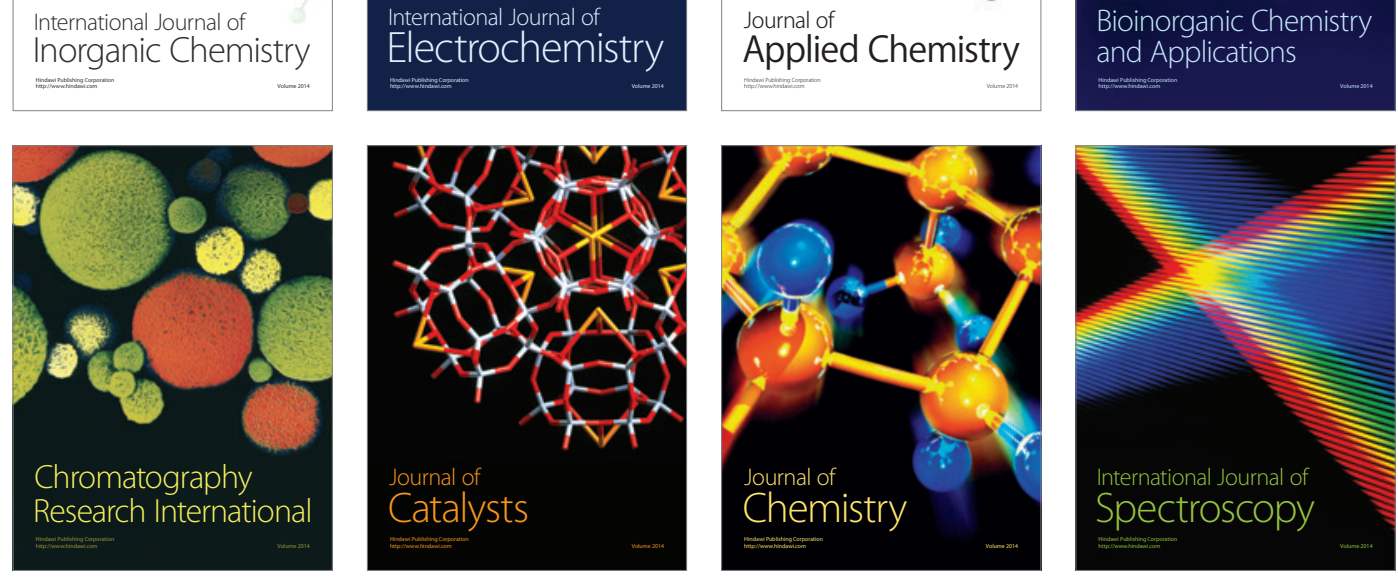